\title{
riccafd
}

Revista Iberoamericana de Ciencias de la Actividad Física y el Deporte

\section{ENSEÑANZA-APRENDIZAJE EN LA CLASE DE EDUCACIÓN FÍSICA}

\section{TEACHING-LEARNING IN THE PHYSICAL EDUCATION CLASSS}

García Peña, M.A. ${ }^{1}$

${ }^{1}$ García Peña, M.A. Licenciado en Educación Física. Docente, Magíster en Salud Colectiva de la Universidad de Antioquía. Instructor Servicio Nacional de Aprendizaje (SENA). Medellín. Colombia. magape68@gmail.com

Código UNESCO: 5801 Teoría y Métodos Educativos

Clasificación Consejo de Europa: 5 Didáctica y metodología

Recibido el 17 de noviembre de 2017

Aceptado el 3 de febrero de 2018

Correspondencia:

García Peña, M.A. magape68@gmail.com

DOI:http://dx.doi.org/10.24310/riccafd.2018.v7i3.5537

\section{RESUMEN}

Analizar y comprender los componentes del proceso de enseñanza-aprendizaje (E-A) en la clase de Educación Física realizada entre docentes y escolares de una institución educativa de Medellín, es la intención de esta investigación cualitativa y con el enfoque etnográfico particularista, permitió evidenciar a través de las voces de los participantes sus percepciones sobre el tema en cuestión. La información obtenida se recolecto a través de las técnicas investigativas tales como: Observación participante, entrevistas y talleres.

El análisis se realizó bajo codificación abierta y axial, en la categoría de la metodología del proceso (E-A). Como resultado de la indagación, quedo claro que en la clase de Educación Física se concreta el quehacer docente, entendido como una integralidad entre saberes y vivencias, donde el quehacer debe tener: un inicio -la planeación, un desarrollo- las estrategias de enseñanza aprendizaje y un final- la evaluación.

Los momentos y espacios para la planeación deberían ser de acuerdo a los objetivos y necesidades de los escolares establecidos en un diseño curricular, durante el desarrollo de la clase el docente debe tener claro los objetivos planeados, cuales debería ser sus herramientas metodológicas para la orientación adecuada y pertinente de la asignatura, y para finalizar el proceso formativo, es pertinente clarificar los elementos desarrollados en la sesión que serán 
valorados al finalizar cada encuentro, que sirven de insumos para el momento evaluativo.

Los hallazgos en este trabajo dan cuenta de visiones parcializadas durante la construcción de la planeación como su ejecución, así mismo de las diferentes formas de evaluar los procesos, lo que deja entrever una nueva indagación hacia el desarrollo metodológico de la enseñanza.

Palabras clave: Educación Física, metodología, practicas pedagógicas, enseñanza-aprendizaje, evaluación.

\section{ABSTRACT}

Analyzing and understanding the components of the teaching-learning process (E-A) in the Physical Education class carried out among teachers and schoolchildren of an educational Institution in Medellin, is the intention of this qualitative research and with the particularistic ethnographic approach, it allowed to demonstrate through from the voices of the participants their perceptions about the subject in question. The information obtained was collected through investigative techniques such as: Participant observation, interviews and workshops.

The analysis was carried out under open and axial coding, in the category of the process methodology (E-A). As a result of the inquiry, it became clear that in the Physical Education class the teaching task is concreted, understood as an integrality between knowledge and experiences, where the task must have: a start-planning, a development-teaching-learning strategies and an endevaluation.

The moments and spaces for the planning should be according to the objectives and needs of the students established in a curricular design, during the development of the class the teacher must have clear the planned objectives, which should be his methodological tools for the adequate orientation and pertinent of the subject, and to finalize the formative process, it is pertinent to clarify the elements developed in the session that will be valued at the end of each meeting, which serve as inputs for the evaluative moment.

The findings in this work account for biased visions during the construction of planning as its execution, as well as the different ways of evaluating the processes, which suggests a new investigation towards the methodological development of teaching.

Keywords: Physical Education, methodology, pedagogical practices, teachinglearning, evaluation. 


\section{INTRODUCCIÓN}

La educación es una cuestión ética y de compromiso social y no sólo debe mirarse como un proceso metodológico transmisor de conocimientos desconectados del contexto (1) por tanto, comprende un proceso complejo que permea la vida y el desarrollo del ser humano. Así mismo, desde la concepción (2) la educación debe ser una práctica movilizadora.

Un aspecto central, es reconocer que la educación se da en todos los escenarios donde transcurre la vida, sin embargo, la escuela es privilegiada para sus fines porque concentra sus acciones en procesos de aprendizaje socio-vivencial, al motivar la construcción de relaciones con diversos elementos que implican el aprendizaje de normas, negociaciones, las cuales potencian el ejercicio democrático (3). De esta manera, en la escuela mientras van y vienen áreas del conocimiento, se deja de lado un aspecto fundamental que debe plantearse en las discusiones al respecto y es la formación integral para la vida, para el desarrollo y el despliegue de potencialidades y capacidades humanas (4). Los docentes, cualquiera que sea su área de conocimiento, deben inculcar actitudes y valores que faciliten el proceso de socialización del escolar y permitan un consecuente desarrollo (5).

En este sentido, un área que aporta al desarrollo integral en este campo es la Educación Física, considerada: «como el arte, ciencia, sistema o técnica de ayudar al individuo al desarrollo de las capacidades físicas» (6) y de interacción con la vida que implica destrezas, armonía de movimientos, agilidad.

Otros autores plantean la labor de ayuda, uno de ellos es (7) quien la define como: "la ciencia y arte de ayudar al individuo en el desarrollo intencional de forma armoniosa, natural y progresivo, de las posibilidades de movimiento personales» (p.18), acentuando la consideración del movimiento humano como agente pedagógico de la formación integral.

Allí, en la Educación Física como en todas las áreas de conocimiento hay elementos constitutivos del proceso de formación integral y son las prácticas pedagógicas consideradas (8), como procesos de cambio que deben incidir en la forma de construir elementos del conocimiento y de propuestas educativas significativas. Por lo tanto, es propicia la invitación que éstas se desarrollen a través de la planificación, la estructuración metodológica de contenidos de la enseñanza, las interrelaciones presentes entre docente y escolares, en torno a las actividades académicas. Permiten también, la orientación adecuada del proceso educativo y jalonan el fortalecimiento de los procesos educativos en el área y la implementación de nuevas estrategias, en la enseñanza.

Así, los procesos pedagógicos que ocurren en estas prácticas, se caracterizan por ser abiertos y complejos, en los cuales el profesor es un actor que gestiona las situaciones que se presentan. De allí, que la comprensión y sentido de las prácticas pedagógicas debe ubicarse en los vínculos entre las acciones rea- 
lizadas, sus objetivos y sus efectos, las cuales deben estar cargadas de sentido para mejorar y modificar los resultados de los escolares (9).

La práctica de la Educación Física, en su espacio escolar, está atravesada por varios elementos, entre ellos, la motivación del docente para acompañar y enseñar el acto educativo y la de los estudiantes por aprender, para mejorar la interacción durante el proceso enseñanza-aprendizaje. Igualmente, las metodologías que son el vehículo por medio del cual se logran los objetivos pedagógicos y su aporte al desarrollo de habilidades para la vida y destrezas de los escolares (10).

El área de la Educación Física, desde algunas miradas, es asumida como una labor técnica, la cual se sustenta en una postura corporal militar y un adiestramiento del cuidado del cuerpo por prescripción (11). De igual forma en la clase de Educación Física se identifica un alto sentido deportivista (12) que, a su vez, con sus diferentes formas de ejecución, puede contribuir de forma positiva o negativa en la salud física, mental y social y la interacción, ya que la voz del docente se asume como un dispositivo de poder (13).

De esta manera es necesario dar una mirada a las prácticas pedagógicas en la educación, puesto que son un campo complejo que requiere según Bruner, (14) ahondar en el proceso de enseñanza aprendizaje, que considera como: "la caja negra» a la que no se toca. Esto también lo retoman (15), en sus estudios del área de enseñanza, al establecer que es necesario, en América Latina, indagar sobre las propias prácticas para la construcción de conocimientos que permitan conocer lo propio y evitar importar metodologías foráneas, las cuales no siempre se ajustan a las realidades del contexto al que se aplican (15). Asunto que perfectamente aplica para la Educación Física, que no escapa de estas formas de investigación que dejan por fuera las prácticas pedagógicas. En este sentido, dicho estudio que es para Chile, puede traspalarse analíticamente al contexto colombiano, el cual ha sido también afectado por la colonización de modelos pedagógicos, no convenientes a nuestras necesidades y realidades.

Debe entonces pensarse y repensarse si lo que se está haciendo con la Educación Física, apunta a objetivos de tipo deportivistas; o, por el contrario, genera y promueve una actividad física para la vida. Por su parte los docentes deben avanzar en pensar su profesión en términos de ser educador físico o seleccionador deportivo y dimensionar la importancia y relevancia de emprender una educación desde la base, evitando desvíos del que hacer del acto pedagógico.

Por los elementos presentados anteriormente, se desarrolló la investigación: Significados de las prácticas pedagógicas de la clase de Educación Física que construyen docentes y escolares en una institución educativa de Medellín, 20152016, la cual se orientó a comprender los significados de las prácticas pedagógicas para docentes y escolares y en ello, una categoría central se orientó a las estrategias de enseñanza aprendizaje que se utilizan en las clases. Y precisamente por el interés de visibilizar parte de los hallazgos, se presenta este artículo que da cuenta de algunas reflexiones en términos de la metodología. 


\section{MÉTODOS}

Es una Investigación cualitativa con perspectiva metodológica etnográfica, es decir, un estudio alrededor de la conducta humana y de los significados sociales incorporados en los espacios educativos en este caso. Se soportó en las ciencias sociales, por ello, es un producto social atravesado por valores, percepciones y significados que se construyeron en el ir y venir durante el proceso (16) y referido en su más amplio sentido, a la producción de datos descriptivos, teniendo en cuenta las propias palabras de las participantes, tanto escolares, como docentes, habladas o escritas y la conducta observable. Se siguieron los lineamientos de la Etnografía enfocada (17) la cual se centró en describir en detalle un fenómeno particular, como fueron las prácticas pedagógicas de la Educación Física, en un escenario concreto que es una institución educativa en la ciudad de Medellín.

El acceso al campo se realizó posterior a los avales del Comité de Ética de Investigación de la Facultad de Enfermería, mediante el contacto con los directivos de la institución Educativa, quienes remitieron a los profesores del departamento de Educación Física. Durante el trabajo de campo, se permaneció en contacto con los participantes, quienes estuvieron ampliamente informados de los avances de la investigación. El trabajo de campo fue permanente y se definió salir del campo al tener los insumos, que daban cuenta del cumplimiento de los objetivos de la investigación.

Los participantes en este estudio fueron 9 docentes y 14 escolares, quienes se incorporaron a partir de un muestreo por intención subjetiva o conveniencia, basados en las orientaciones de autores, (16 y 18) teniendo en cuenta los objetivos de la investigación, la disponibilidad del tiempo de cada uno de ellos y las posibilidades individuales para los encuentros, ello permitió dar cuenta del fenómeno u objeto de estudio.

Las técnicas utilizadas fueron observaciones y entrevistas.

Las observaciones (19), se concertaron con los participantes y se realizaron por 4 meses en diferentes escenarios y durante el tiempo de la clase. El trabajo se centró en las interacciones entre los participantes y en la metodología.

La entrevista semiestructurada (20), se desarrolló con los docentes, en el tiempo pactado con cada uno de ellos, en un ambiente cómodo y propicio que favoreciera la comunicación.

Para el abordaje de los estudiantes se realizó una entrevista estructurada, ya que implica la aplicación sistemática de unas mismas preguntas, es rígida, definida y limita el nivel de profundidad (21). Posterior, se realizó un taller por cada jornada, (mañana y tarde) siguiendo el objetivo de identificar motivaciones en la participación de la práctica de la Educación Física. 
La información se recolectó hasta lograr el límite de saturación de la misma, (19) es decir, hasta notar que en la información obtenida se mantenían patrones de repetición y no aparecían nuevos elementos, a lo que se sumaron, los límites de tiempo.

Para el proceso de análisis de los datos derivados de los diarios de campo, las observaciones, las entrevistas y el taller, se trascribió textualmente la información obtenida en texto de Word, posteriormente se codificó con el Software de análisis cualitativo Atlas ti 6.2, siguiendo las categorías sensibilizadoras de: 1) motivaciones 2) metodología y 3) significados.

Hubo inicialmente una lectura general para identificar temas comunes, luego se realizó la codificación, conformación de categorías y subcategorías. Se llevaron las categorías con sus textos significativos a una matriz en Excel y a mapas que facilitaron la organización y escritura descriptiva del sustrato que generaba la información.

En cuanto a los significados de las prácticas pedagógicas, los docentes se orientaron a la valoración, y el reconocimiento del otro; mientras que para los escolares lo que significa la clase de educación, tiene que ver con los momentos de práctica deportiva o de recreación, así como el gusto por ejercitar el cuerpo, que se relaciona con la mente, la salud y la vida. En las motivaciones emergieron las interrelaciones durante la clase de Educación Física, de docente-escolar y escolar-escolar; además, con los escolares se dio un acercamiento hacia el gusto por la clase y su importancia para la vida. En la metodología se trabajó todo lo concerniente a las subcategorías de la planeación, la evaluación y el proceso de Enseñanza -aprendizaje (E-A).

Esta investigación sigue los criterios de estudios cualitativos los cuales son: la credibilidad, la auditabilidad y la transferibilidad, (22) que son básicos en el procedimiento, para asegurar la calidad (23).

Se enmarca en las normas científicas, técnicas y administrativas asunto que tiene que ver con la investigación en salud, establecidas en la Resolución 008430 de 1993 del Ministerio de Salud de Colombia (24), según la cual no tuvo ningún riesgo, pues, no se realizó intervención o modificación intencionada de aspectos psicológicos o sociales de los participantes. Además de las consideraciones del Consejo de Organizaciones Internacionales de las Ciencias Médicas (25), el respeto (principio de reciprocidad) por los participantes, se generó un ambiente de bienestar durante las interacciones. También se salvaguardó su intimidad, no se presentaron sus nombres en los resultados. De igual forma el trabajo contó con el consentimiento informado para docentes y padres de familia, así como con el asentimiento de los escolares.

Este trabajo siguió las indicaciones del Comité de Ética de Investigación de la Facultad de Enfermería de la Universidad de Antioquia. (Acta N CEI-FE 201527 - 28 de agosto de 2015). 


\section{RESULTADOS}

Según los datos que arroja el estudio, en la clase de Educación Física como escenario de la práctica pedagógica, la metodología de trabajo con los escolares es esencial, porque concreta en el quehacer del docente, en el orden de la clase que se nombra como un viaje a la práctica debido a que tiene un inicio = planeación, un desarrollo = proceso de enseñanza y de aprendizaje $(E-A) ; y$ un final = evaluación.

\section{Trazando el norte}

Como lo plantearon los docentes, la planeación juega un papel importante en el desarrollo de la práctica pedagógica de la Educación Física, puesto que es allí donde se traza el norte, se fijan las estrategias para fortalecer y llevar a cabo con los escolares el desarrollo de las habilidades y las destrezas, los valores, los conocimientos y la interrelación con los otros participantes de la comunidad educativa.

Así, se parte de algunos encuentros de trabajo de los docentes del área de Educación Física sobre el desarrollo curricular. Allí, se realiza la planeación teniendo en cuenta los contenidos del área, los objetivos, y las estrategias para llevar a cabo la clase de Educación Física, como lo expresa un participante: "dentro de las cosas fuertes que ha tenido la Institución, ha sido ese tipo de planeaciones, el dar un espacio especial, tal vez son de las pocas instituciones que tenemos un espacio especial los días viernes para reunirnos [...] y poder hacer esas planeaciones» E5H57DV. En estas se plantean y ofrecen una gama de actividades académicas, de uso del tiempo libre y de interrelación con sus pares, con la oportunidad desde lo deportivo para ejecutar y fortalecer las habilidades físicas y deportivas.

La carga docente implica el desarrollo de múltiples actividades, hay momentos en que la planeación es individual y no permite la socialización pertinente para su desarrollo: "realmente nosotros planeamos determinadas actividades muy solitarios por la misma cantidad de actividades que tenemos y muchas veces no las socializamos» E5H57DV.

Se reconoce que el proceso educativo, depende inicialmente de la planeación, la cual permite con una orientación establecida, el logro de unos objetivos claros y pertinentes, teniendo en cuenta: ¿qué es lo que se quiere?, ¿qué se pretende alcanzar?, y ¿qué metodología aplicar para el mejoramiento de la calidad educativa?

\section{En el proceso se enseña y se aprende}

Enseñar y aprender (E-A) se da entre los actores: docentes y escolares. EI docente responsable de llevar a cabo la clase con una adecuada planeación, no sólo de unos temas deportivos-corporales, sino con el compromiso de reafirmar 
valores, fortalecer los comportamientos y desarrollar habilidades y destrezas motrices en los escolares. En el proceso de E-A en esta institución educativa hay dos grandes orientaciones, una deportivista y la otra, predeportivista. Allí se desarrollan algunas estrategias didácticas para el desarrollo de la clase, que tienen que ver con el mando directo, la asignación de tareas, el manejo de las técnicas deportivas, el juego y los talleres teóricos, que, en su conjunto, permiten a los escolares el desarrollo propio del área de la Educación Física.

La orientación deportivista se da en los grados superiores: «la orientación es muy deportivista porque tenemos buenos escenarios deportivos, entonces, siempre estamos buscando eso, llevar al alumno al deporte, [...]» E7H52DV, mientras que en los grados inferiores prima un enfoque pre deportivas y de desarrollo de habilidades: "en primaria se trabaja el deporte como una actividad pre deportiva, sobre todo que los niños son muy competitivos también, pero intentando pues que sea más recreativa», E1H46DV, y al desarrollo de las habilidades y destrezas de los escolares: "todo lo que hace con los niños es básicamente como desarrollar las habilidades, destrezas, donde ellos puedan pues obtener mediante el trabajo las capacidades como pueden recibir un balón o como lanzarlo, como saltar, como correr, o sea enseñarle estas actividades básicas» E1H46DV.

De igual forma, otro elemento constitutivo del proceso es el método, el cual se particulariza según las características de los escolares: "eso depende [...] de la situación que se nos presente, con algunos alumnos hay que tener un método, con otros otro» F2E4H63DV, por lo tanto, adaptan y adecuan sus formas de enseñar a las necesidades de cada situación. Estos métodos según los docentes pueden ser inductivo o deductivos como lo menciona un docente: « $A$ ver, uno muchas veces utiliza el método inductivo-deductivo, [...] si es un grupo que va bastante avanzado, uno utiliza ya la parte deductiva y ya arranca, [...] esto es lo que tiene que hacer y listo; pero si es un grupo que está empezando, uno tiene que empezar, la parte inductiva, de lo menos a lo más» F2E5H63DV. Para los docentes lo inductivo lo definen como: ir de lo fácil a lo complejo.

Entonces, cada docente le imprime su sello personal al trabajo para orientar o dirigir la clase y lo hace diferente de sus pares: "yo dentro de mis clases periodo a periodo, trato de sacar unas clases teóricas cierto, donde se les muestra mediante videos, mediante algunas charlas, de algunos talleres la importancia de la Educación Física cierto» E1H46DV, lo que permite aplicar en cada clase diferentes métodos de trabajo: como el constructivista o el tradicional, o en ocasiones ambos. Sin embargo, se puede orientar la misma actividad de formas distintas, que marcan esa diferencia en el proceso educativo de acuerdo con los grupos y los grados.

También, es común que cada docente de Educación Física tenga un protocolo como requisito básico para llevar a cabo la orientación de la clase en el proceso educativo, por lo tanto consta de un orden o secuencias de tareas, entre ellas una fase inicial, una central y un final o vuelta a la calma: "calentamiento, parte central y fundamentación, que haya coherencia de la temática con las actividades a realizar, que tenga sentido»[...], Entonces para mí eso es supremamente 
importante, y que otra intención, que estén muy bien, que se sientan a gusto con la clase. F2E2M41DV. En este proceso, algunos docentes consideran que fueron formados en conceptos viejos, siguen haciendo en su clase lo mismo del pasado: "somos maestros formados en conceptos viejos y no hemos podido cambiar todavía la Educación Física, seguimos reproduciendo lo mismos de hace veinte, treinta años» E7H52DV.

Además de lo anterior, otro elemento que hace parte de la E-A, son las estrategias que orientan la clase, entre ellas, se destacan: la asignación de tareas, el mando directo, la explicación de técnicas, el juego y los talleres teóricos, como estrategias evaluativas.

Otra estrategia, es el mando directo, que es muy reiterado y se realiza con el grupo en pleno. La instrucción es dada de manera general y supone el cumplimiento de la actividad por parte del escolar, por ejemplo dice el docente: «elevación de rodillas, ahora brazos, luego hacemos flexiones de pecho, [...] O3H50DV, de nuevo «vuelta a la cancha, a trotar caballeros, vamos por favor a calentar - trotando pues que no es una procesión» O8H55DV, son así las instrucciones permanentes para la ejecución de las actividades de diferentes maneras: «El trabajo que se va a realizar se hará en parejas, vamos a calentar, listos vuelta a la cancha, luego vamos a estirar» O10M41DV.

Para el manejo de la técnica, la interacción entre escolares es muy importante puesto que uno enseña al otro: "en ese momento una de sus compañeras se arrima y le indica cómo debe recibir el balón (gesto de antebrazos) para no ser golpeada con el balón» O8H55DV. También en el manejo de la técnica se utiliza la demostración que consiste en que el docente hace o plantea un ejercicio, gesto, etc., y el escolar lo realiza o imita: «Bueno ehhh, la verdad pues a veces hay estudiantes que son modelos dentro de la clase, cierto, entonces con ellos ehh hacemos un modelo de demostrar para ellos vean cómo se hace la actividad [...]»F2E1H50DV.

También, el juego permite la interiorización y reflexión de lo realizado en la práctica durante la clase de Educación Física: «Mi clase es prácticamente hacer ejercicio, moverse [...] entonces el juego es fundamental pues desde la recreación, el juego es fundamental. Utilizo también mucho la reflexión, trato de que ellos siempre ubiquen por qué fue importante hacer esa actividad. No me quedo solamente pues con el hecho de practicar, claro pues ellos dicen de una, si muy bueno. Pero llevarlos al hecho de que reflexionen que fue lo que se hizo ahí, interiorizan un poco más la práctica que hicieron» F2E2M41DV.

Así mismo, se propende por un juego que permita un espacio de alegría, aprendizaje e interrelación, que su vez permita el desarrollo de habilidades según el nivel de los escolares.

Otra estrategia utilizada son los talleres, los cuales se implementan con escolares que presentan algún tipo de incapacidad médica, dificultad física o discapa- 
cidad. Estos buscan dar cuenta del interés escolar por el área, su compromiso y aportes a los conocimientos de algunos temas, como la prevención de lesiones osteomusculares, técnicas de manejo deportivo, entre otros, que posterior a su entrega, son socializado con sus pares, y evaluado por el docente, así como se indica: «dentro del grupo de trabajo de la institución del área tenemos unos talleres. Si un estudiante, que por decir algo estamos en futbol y presenta incapacidad para estar en futbol, en muchas clases de trabajo debe entregar un trabajo escrito de ese deporte» E1H46DV.

Puede considerarse que las estrategias mencionadas corresponden con el método tradicional en el que el docente dice y el escolar hace, aquí se dejan por fuera el desarrollo de otras habilidades de los escolares. Sin embargo, hay algunos docentes que trascienden estas prácticas y se enfocan en estrategias vinculantes que propenden por la autonomía, el desarrollo de los sentidos, y la posibilidad de que se realicen con ganas las actividades: «Pienso que la mayor estrategia es hacer las cosas con muchas ganas, con mucho amor, con mucha disposición y traer la clase bien planeada, bien organizada de manera que uno sepa que a ellos les agrada, porque a ellos les encanta es la actividad, les encanta eso» E6M37DV.

Este sería el ideal y el compromiso serio de todos los que están implicados en el proceso y divulgan la Educación Física, pero debe haber un compromiso real en la edificación y desarrollo de estudiantes integrales, donde primen los intereses y las necesidades de los mismos, traspasando los diseños curriculares tradicionalistas de valorar sólo contenidos y aptitudes físicas, sin tener en cuenta una verdadera y real valoración del ser.

\section{Dando valor a lo hecho}

La evaluación como parte del proceso educativo de la enseñanza procedimental y sistemática dentro de sus fines, busca estimular la formación integral de los escolares, a través de la valoración del desarrollo de competencias para el conocimiento y el desempeño armónico, para el alcance de los objetivos planteados en el Proyecto Educativo Institucional (PEI). Tal valoración es hecha por los docentes y se plasma con una nota que determina si cumplió o no los objetivos del área. Para el proceso de evaluación, en la I.E. se tienen en cuenta las orientaciones del Ministerio de Educación Nacional de autoevaluación, coevaluación y heteroevaluación y aparece consignado en el Manual de Convivencia de la I.E. (26).

En la autoevaluación el escolar tiene la oportunidad de asumir su proceso de aprendizaje y a partir de allí darse una nota; frente a esto, un docente expresa: «bueno muchachos ya solo faltan dos clases [...] van a darme una nota de ustedes a través de una autoevaluación de mini-baloncesto» O10M41DV.

De la coevaluación referida al desempeño grupal colaborativo se infiere la valoración que el escolar hace de la clase: «Para mí es más importante que ellos me digan cómo, como se sintieron. Nosotros aquí tenemos una estrategia que, 
que me parece supremamente válida que es la coevaluación, entonces unas les establece unos criterios a ellos, 5 o 6 criterios [...] entonces eso me parece que los va a llevar a ellos a pensarse en lo que hizo, que se obtuviera o no conocimiento, y que ellos pudieran dar un aporte puntual al aprendizaje» F2E2M41DV. Mientras que la heteroevaluación implica la valoración desde el docente, que es la que más destaca en este estudio.

Para algunos docentes la evaluación de su curso tiene que ver con su satisfacción, es decir que evalúan según el cumplimiento de sus expectativas, tal como lo indica un profesor: «A veces cuando no me siento conforme los evaluó y lo repienso. De resto casi no hago evaluaciones de mis clases, trato de actuar y desempeñarme lo mejor allá, a estas alturas ya uno no evalúa» E7H52DV.

Una pretensión de la evaluación es que sea integral, para ello es fundamental considerar algunos elementos desde lo personal y lo colectivo. Lo primero: el cumplimiento, la responsabilidad, la puntualidad, la asistencia, el interés, la disciplina, la participación, la disposición, el desarrollo motriz, los conocimientos de los temas asignados, las ganas de hacer las cosas tal como lo refiere un docente: «Me limito a evaluar el interés, la motivación, el deseo. Mientras tengan el deseo y las ganas de aprender, con seguridad las notas van a ser siempre positivas, cuando no hay esos elementos la nota no da, es tanto, ni el hecho que hagan o no un movimiento correcto o incorrecto no es significativo, es más significativo el deseo, las ganas de aprender por lo menos la disposición, cuando los alumnos no manifiestan eso, no hay nada que hacer" E2H53DV.

En segundo lugar, se destaca que los escolares tengan una participación activa a través de trabajos grupales, la interacción con los otros, práctica de juegos deportivos y pre deportivos; tareas que permitan realizar el seguimiento y la evolución de lo enseñado en la clase: "Seguimiento de cada clase, asignando códigos que me permiten visualizar el comportamiento en general. Evaluaciones prácticas de uno o varios aspectos de la técnica que en general aprendieron. Talleres grupales que permitan evolucionar lo aprendido en clase» F2E6H55DV.

Con la evaluación en términos de una nota, se busca mejorar unos resultados académicos y esto es lo más importante en los escolares, como lo refiere un docente: "trato es de que ellos obviamente entiendan que la nota no es lo más importante pero, entonces hay yo me pongo un poco drástico en la nota» F2E1H50DV, también la nota se relaciona con cumplir exitosamente un ejercicio según las indicaciones docentes, sin embargo hay unos límites que llevan a la nota satisfactoria: "también que haga las diez cestas [es difícil], cinco, de ahí para abajo se calificaba. Ya uno no puede hacer, porque eso ya es difícil, y así perderían más estudiantes» E4H56DV.

Un punto crítico que destacan los docentes es el sometimiento a la nota que hace que los muchachos estén pendientes de la misma: "yo soy consciente, la actividad física dentro del sistema educativo nos somete a sacar una nota, a es- 
tar inmersos o estar pensando siempre en la nota y el muchacho piensa siempre en la nota y no hemos podido sacarlos de ahí [...]» E7H52DV.

El proceso evaluativo durante la clase se vuelve complejo, cuando no se tienen objetivos claros, puesto que ellos vienen establecidos desde la planeación curricular; sin embargo, se tiende a evaluar elementos de carácter actitudinal y disciplinarios a la hora de emitir una valoración, que se refleja en una nota; contradictorio al planteamiento último, de no sacar a los estudiantes de estar pendiente de la nota, que en últimas instancias dice si gana o no en cada período académico.

\section{DISCUSIÓN}

Debido a lo presentado en términos de los resultados, se exhiben dos tensiones fundamentales: 1) la exigencia del cambio hacia didácticas activas a unos profesores formados en conceptos tradicionalistas que siguen reproduciendo; 2) el salto de la evaluación como proceso de control a la evaluación como proceso de formación integral.

En lo que respecta a la exigencia de didácticas activas a los profesores formados en la vieja escuela, es claro que la educación actual, requiere de nuevas estrategias metodológicas para la enseñanza de las diversas áreas del cocimiento. Son bastantes los estudios que ofrecen alternativas, sin embargo, la actualización y cualificación del personal exige mayor compromiso, tanto de los centros educativos de la formación, como de los nuevos educadores. Un mercado que exige mayores compromisos y retos, ante una sociedad cada vez más exigente, pero que también se ve limitada por lo fácil y lo de mínimo esfuerzo.

Los cambios en el contexto han aumentado las exigencias a los docentes: a obtener mayores responsabilidades frente a los conocimientos, a los valores que deben tener para el desarrollo del trabajo con los estudiantes, al manejo de nuevos métodos para la enseñanza con respeto a los contenidos, aumentar las competencias para interactuar con la sociedad, involucrar las técnicas que se relacionan con los avances tecnológicos, introducirse al campo de la investigación y la reflexionar sobres sus prácticas (27).

En esta misma línea, el estudio (28) revela que las nuevas competencias y demandas profesionales aventajan a los saberes tradicionales, incluso la planificación y las estrategias de enseñanza, sin embargo, parecen estar en regresión. En este sentido las instituciones en su mayoría idealizan a los docentes para dar los contenidos de enseñanza, pero en ocasiones estos no saben cómo organizar y orientar una clase (29).

Asimismo, las exigencias no pueden quedarse en la formación tradicional, por el contrario, pueden ser una oportunidad en aplicar diferentes estrategias que permitan darle una orientación adecuada a la clase, en este caso la clase de Educación 
Física, evitando la monotonía, la repetición de ejercicios, etc. Esto permite cuestionar como se sigue llevando a cabo el direccionamiento de forma tradicionalista en la clase, pese a que hay unos modelos de enseñanza alternativos propuestos, en la institución que sustentan que debe ser diferente. Así mismo algunos docentes tienden a caer o seguir en la repetición en términos de contenidos.

El autor (30) plantea que la repetición mecánica de lo dicho por el docente, o de un libro u otro recurso, no permite avanzar en términos de la formación crítica y creativa, porque el estudiante no es un producto acabado y por ello se debe promover el desarrollo de éstas capacidades para esta nueva época, como estrategia para trasformar los conocimientos y evitar caer en el tradicionalismo

Debe considerarse, además, que las acciones repetitivas en las clases de Educación Física «siempre lo mismo», son fuente de desmotivación y deseos de no hacer nada; a la vez, son un pretexto con el que se escudan los estudiantes para no asistir a clase; pues es predecible lo que pasará en tanto habitualmente en ella se repiten las mismas cosas y de la misma forma. Por lo general, los estudiantes de antemano ya saben cuáles son las fases y el tipo de actividades para realizar en la clase. Lo anterior se debe en gran medida a que el docente considera que no necesita planear las clases porque domina los contenidos, la metodología y las formas de organización de los escolares (31). Tales asuntos se corresponden además estudios (32) dan cuenta de las excusas para no participar o asistir a la clase, todo por la repetición de las mismas cosas.

Estas son debilidades fuertes en el trabajo, por lo que debe tomarse como una oportunidad para la implementación de la participación permanente en la construcción de unas prácticas de la actividad física con base en las necesidades e intereses de los estudiantes y en la que la Institución permita disminuir la rigurosidad de los lineamientos y requisitos estandarizados establecidos por el sistema educativo, para que se vea lo misional, lo de una educación centrada en el ser humano, sin embargo, en la realidad, no se alcanza dimensionar.

En el planteamiento (33) es que la enseñanza no puede centrarse en repetir solamente información, por el contrario, es permitir experiencias de aprendizaje significativas, donde se adquiera no sólo esa pesquisa, sino que trascienda y se manifieste en el comportamiento, en sus emociones, en la capacidad crítica; aportando al desarrollo de sus habilidades para el dialogo, la participación y la tolerancia frente a sus compañeros.

Cabe entonces pensar que los currículos deben estas enmarcados en formar personas propositivas, reflexiva y críticas, dónde los docentes saquen a sus estudiantes del encierro intelectual y puedan confrontar lo vivido en la clase con su entorno, se arriesguen a descubrir su esencia, y puedan construir una sociedad más objetiva, (34). Es decir, que el escolar como ser humano, a partir de su proceso formativo, adquiera herramientas para la vida, que le permitan construir un criterio autónomo e íntegro, puedan tomar postura frente a la vida, aunque esto se complejiza, cuando emprende la interacción con otros. 
En los encuentros de los docentes se debe estructurar todo el engranaje educativo, a través de la planificación curricular con las temáticas acordes para las clases; donde se socialice y se den a conocer las características y las necesidades individuales y grupales de los estudiantes, lo que implica el diseño de las diferentes estrategias de E-A, la adecuación de los espacios, la elección de los objetivos, el diseño de estrategias motivacionales, al igual que las de control y seguimiento al proceso, la utilización de fuentes de información y la actualización de temas relacionados, para impulsar la participación y la interrelación con y de los estudiantes. Estos elementos permiten fortalecer los procesos educativos y el desarrollo de los mismos, todo en pro de mejorar las dinámicas de trabajo y la trasformación de los procesos educativos.

Por todo lo anterior planteado, el docente debe ser un enamorado de su profesión, comprometido con la vocación como un don privilegiado, que lo hace saber que al frente tiene un potencial y que, a través del acto educativo de la Educación Física, puede transformar y enseñar a ver de forma diferente a la vida. Además, tiene en sus manos la posibilidad de cambiar a muchos estudiantes con el solo hecho, de saber que ellos pueden pensar diferente, utilizando su mejor estrategia didáctica: «el amor», para sensibilizar y humanizar a la educación.

Las prácticas pedagógicas, con las diferentes áreas del conocimiento, pretenden aportar a los procesos educativos, a través de la enseñanza-aprendizaje, (2): «no sólo enseñar los contenidos, sino también enseñar a pensar correctamente», por ello, es relevante repensar qué es lo que se pretende educar en nuestra sociedad: si es seguir adiestrando seres humano no pensantes y dar cumplimiento a normas de cantidad, mas no de calidad; o materializar las posibilidad de humanizar a la educación.

Se menciona (31) que la planeación es un requisito administrativo, ayuda a la organización de las actividades y el logro de los objetivos, facilitando la tarea del docente, utilizándola como un recurso pedagógico; sin embargo, se sigue presentando la falencia en el tema. Por lo que se pudo establecer en el trabajo de campo se continúa con formas de trabajar la clase de Educación Física mecánicamente, donde el estudiante hace o repite las tareas asignadas por los docentes, quienes orientan, controlan y verifican lo planteado, para luego trascribirlo a una planilla, y hacer su propia evaluación de acuerdo con los criterios establecidos, como se menciona más adelante, como un proceso simplemente de control.

Con todo lo anterior, el trabajo permitió vislumbrar una debilidad: la falta de didácticas activas para la orientación de la clase, las actuales requieren de actualización de los procesos metodológicos y que pueda servir de derrotero para los docentes.

La tensión del salto de la evaluación como proceso de control a la evaluación como proceso de formación integral, como parte del proceso educativo; no deja de ser tema de estudio permanente en todo el acto que realice el ser humano, la dife- 
rencia radica en qué y por qué se evalúa: para mejorar procesos, para dar una validación de algo, para cuantificar, para cualificar; serían muchas las razones, pero la que nos compete, en la práctica pedagógica, es el acto mismo de la formación.

Cabe preguntar: formar ¿para qué?, por lo que la evaluación requiere de un acto reflexivo permanente, ya que en ella se juegan diferentes intereses, desde las instituciones formativas, con sus docentes y escolares como los estudiantes protagonistas del acto educativo. De igual forma padres de familia, quienes desean tener resultados positivos de sus hijos, ante una sociedad que impulsa cierto tipos o estilos de comportamientos dentro de la cultura.

Se convierte así, la evaluación en un signo compulsivo y de amenaza para los estudiantes, pues para ellos es un mecanismo de presión y desagravio, en donde los docentes asumen ciertas posturas acríticas referentes al acto evaluativo, presentando algunas deficiencias conceptuales y limitaciones para evaluar. Por lo tanto, se perpetúa el autoritarismo desde los sistemas de gobierno y de la sociedad, que viene de los siglos XII y XIII con una filosofía del poder desde la educación escolástica, que aún perdura en nuestra cultura (35). En esta misma vía, hay una evaluación sin criterios por parte del docente en la clase, que se limita a la «nota» o calificación, pero no al proceso como tal. Así cómo se observó en el trabajo de campo.

En el estudio (36) se plantea que la evaluación no responde a los momentos de la clase, es imprecisa y los estudiantes muestran poco gusto y desconocen lo aprendido, por lo tanto, para los docentes hay variedad de iniciativas de criterios que articulen las dimensiones del aprendizaje por la evaluación. En este sentido consideran algunos criterios como: el controlar el uniforme, la asistencia, la participación, el interés entre otros, como elementos de seguimiento y control en los estudiantes.

La evaluación (37) en la educación sigue una orientación tradicionalista que da una calificación al rendimiento educativo, con funciones jerárquicas y de clasificación, siendo así, un instrumento oculto de poder y de control en la clase, generando pánico para no ir a perder el área. Esta, en ocasiones, se convierte en una supervivencia por la calificación, donde el estudiante aprende y resalta que lo más importante es el examen, estudiar por la nota, y desaparece así la motivación intrínseca del aprendizaje y el conocimiento. "La educación deja de ser un proceso de aprendizaje, para convertirse en una carrera de obstáculos que hay que superar» (p.5).

En tal vía, este tipo de evaluación trasgrede el perfil de una educación humanista, generando consecuencias que pueden impedir el desarrollo de los estudiantes y la motivación de y por aprender, se puede ver obstaculizada; por lo tanto es conveniente diseñar instrumentos que contribuyan a estimular el desarrollo de aprendizajes, siendo evaluados de acuerdo con indicadores pertinentes, a las necesidades e intereses, de forma que se personalice la enseñanza, apuntando al desarrollo de habilidades y el de la personalidad (35). 
En el sentido educativo, la evaluación de los aprendizajes por parte de los docentes debe tener un carácter rotundamente formativo, en el que trascienda el saber pedagógico como entidad de discernimiento y se pueda reconocer la comprensión crítica de los saberes en su aplicación. En tal sentido percibir la evaluación como un proceso permanente en la formación, la reflexión, la participación, la cual se centra en el aprendizaje del estudiante y donde el docente es el orientador, canalizador que comprende las dificultades que se le pueden presentar durante el seguimiento del proceso educativo (38).

Es este sentido, la utilización de instrumentos y estrategias que permitan la participación y sensibilización en el proceso educativo; donde los docentes tengan criterios humanistas, eviten ser los sujetos de la evaluación, por el contrario, sean participantes del proceso, orientando la mejora de logros en las conductas, comportamientos y cambios en los estudiantes.

Con lo expuesto, más lo sumado en el escrito del marco institucional, hay una gran controversia entre los hallazgos y lo manifestado por los participantes. Se presenta una ruptura que permite hacer una reflexión frente al tipo de evaluación que se está realizando con los estudiantes, la intencionalidad de los docentes vs la Institución, porque no se puede ir en contravía de lo institucional, puesto que el perjudicado es el estudiante, que en últimas instancias si reclama será calificado, mas no evaluado.

\section{CONCLUSIONES}

La planeación es un componente esencial, especialmente en la educación, donde se forman seres humanos de acuerdo con su contexto, sus necesidades e intereses, por lo tanto, la planeación debe ser proyectada, de manera permanente, hacia: la integralidad, la criticidad, la reflexión en todos los hechos de la vida en la sociedad. Es pieza significativa en el desarrollo y orientación de la práctica pedagógica, donde se establecen y direccionan los contenidos del área, los objetivos, las estrategias y la evaluación, para su ejecución.

El proceso de la enseñanza-aprendizaje debe partir de las insuficiencias de los escolares y de acuerdo con las edades y al desarrollo evolutivo, en la que la planeación es y debe ser el insumo principal. Allí se deben plantear los objetivos, las estrategias y herramientas de la orientación del acto pedagógico y finalizar en la evaluación. Esta debe contener una razón de la valoración del desarrollo y de la ejecución de las actividades, dejando claro el por qué y para qué se evalúa el contenido de cada área de conocimiento.

Las orientaciones de la evaluación desde el MEN en Colombia, autorizada a la I.E, son poco direccionadas hacia los objetivos propuestos, que vienen establecidos con la planeación curricular. Desde allí se tiende a evaluar elementos de carácter actitudinal, disciplinar y contenido, emitiendo una valoración reflejada en una nota, en cumplimiento a una normatividad para proyectar y mejorar los re- 
sultados académicos. Esto constituye un punto crítico: la sumisión (valoración), realizada a los escolares, por lo que la clase se vuelve compleja, cuando no se tienen objetivos claros, no sólo por parte de los docentes, sino desde el mismo sistema educativo.

Por lo tanto, los docentes buscan una integralidad que beneficie más a los estudiantes y consideraran fundamental algunos elementos que definan tanto lo personal como lo colectivo. Destacan: el cumplimiento, la responsabilidad, la puntualidad, la asistencia, el interés, la disciplina, la participación, la disposición, el desarrollo motriz, los conocimientos de los temas asignados, la participación activa, a través de trabajos grupales, la interacción con los otros.

Lo que resalta este trabajo, son las estrategias para la enseñanza de la Educación Física, la utilización deportivista y el método tradicionalista para llevar el acto educativo, por lo que debe ser una educación humanizadora, saludable, que ayude a comprender la cultura del cuerpo y su relación con el entorno, a vivenciar ese cuerpo sin miedo a ser vulnerado, a manifestarse frente a la agresión social, a tratar con respeto al otro, a comprender que su cuerpo habla, siente emociones y sentimientos, vibra con las prácticas deportivas y recreativas.

Tuvo otra importancia agregada, además, porque reveló los vacíos en los procesos metodológicos de las prácticas pedagógicas establecidos desde el sistema educativo, que se manifiestan en desconexión con lo trabajado en la Institución, así mismo, se puede ahondar sobre el tema dedicando mayor tiempo al estudio del mismo, junto con otras áreas del conocimiento y proyectar los tipos de diseños curriculares que aportan a las realidades del contexto.

\section{REFERENCIAS BIBLIOGRÁFICAS}

1. Gonzalez M. La educación Física: Fundamentación y teoica y metodológica. Fundamentos de educación física para enseñanza primaria. 1993; 1.

2. Vidal M, Morales, L. Buenas prácticas docentes. Educación Media Superior [internet] 2009; 23(1): 1-9 [consultado 201705 10] Disponbile en: http://scielo.sld.cu/pdf/ems/ v23n1/ems14109.pdf.

3. Latorre M. Continuidades y rupturas entre formación inicial y ejercicio profesional docente. Revista Iberoamericana de Educación. 2004. Disponible en http://biblioteca. uahurtado.cl/ujah/Reduc/pdf/txt919.pdf.

4. Lucea J. La enseñanza y aprendizaje de las habilidades y destrezas motrices básicas. Inde; 2005; 33.

5. Urrego L. Educación física escolar: el sentido formativo de un área que no cuestiona la tradición. Medellín: Universidad de Antioquia; 2007

6. Benjumea P, Castro J, García C, Trigo E, Zapata M. Desvelando los sentidos de la Motricidad en Colombia. Educación Física y Deporte. Universidad de Antioquia. 2005; 41-63. 
7. Moreno G. (2009). El cuerpo en la escuela: los dispositivos de la sujetación. [Internet] 2009; 9 (1): 159-179. Disponible en www.curriculosemfronteiras.org

8. Brunner M. Educación y escenarios del futuro: nuevas tecnologías y sociedad de la información. Serie Documentos de PREAL 16. 2000.

9. Pasmanik D. Las prácticas pedagógicas en el aula como punto de partida para el análisis del proceso enseñanza-aprendizaje: un estudio de caso en la asignatura de Química. Estudios Pedagógicos. 2005; 31(2).

10. Galeano M. Estrategias de investigación social cualitativa. El giro en la mirada. Medellín: La Carreta; 2004.

11. Boyle J. Estilos de Etnografia. Medellín: Universidad de Antioquia; 2003.

12. Sandoval, C. Investigación cualitativa. Programa de Especialización en Teoría, Métodos y Técnicas de Investigación Social. Instituto Colombiano para la evaluación de la educación (ICFES). Bogotá. 2006.

13. Taylor S, Bogdan R. Introducción a los métodos cualitativos. Barcelona: Paidós; 1992.

14. Valles M. Técnicas cualitativas de investigación social: Reflexión metodológica y Práctica profesional. Madrid: Síntesis Editorial. 2000

15. Tarrés M, Peón F, García R, Wiesner M, Margel G, Gonzalez O. Observar, escuchar y compredenre sobre la tradición cualitativa en la investigación social. El Colegio de Mexico. 2014.

16. Castillo $\mathrm{E}$, Vásquez M. El rigor metodológico en la investigación cualitativa. Facultad de Salud. 2003; 34(3).

17. Lincoln Y, Guba E. Naturalistic Inquiry; 1985; 289-414.

18. Ministerio de Salud; Gobierno de Colombia [Internet] Resolución Nº08430 de octubre 4/1993, por la cual se establecen las normas científicas, técnicas y administrativas para la investigación en salud. Bogotá: Ministerio de Salud. 1993.

19. Organización Mundial de la Salud (OMS). Pautas éticas internacionales para la investigación biomédica en seres humanos. Ginebra: Consejo de Organizaciones Internacionales de las Ciencias Médicas. 2000.

20. Institución Educativa INEM «José Félix de Restrepo». Manual de Convivencia. Ed. Mundo Libro. [Internet] Medellín: Colombia. 2010 [consultado 201708 14] Disponible en: http://www.inemjose.edu.co/.

21. Vaillant D. La identidad docente. La importancia del profesorado. Revista Novedades Educativas. 2010; 234.

22. Alliaud A, Vezub L. El oficio de enseñar. Sobre el quehacer, el saber y el sentir de docentes argentinos. Revista Dialogo Educativo. 2012; 927-952.

23. Esteve J. La aventura de ser maestro. Cuadernos de pedagogía. 2003; 266: 46-50. 
24. Morán P. El reto pedagógico de vincular la docencia y la investigación en el espacio del aula. Contaduría y Administración. [Internet] 2003; (211): 17-30. Institución Educativa INEM "José Félix de Restrepo". Manual de Convivencia. Ed. Mundo Libro. [Internet] Medellín: Colombia. 2010 [consultado 201508 14] Disponible en: http://www.inemjose.edu.co Disponible en http://www.redalyc.org/articulo. oa? id=39521104.

25. Aguayo H. Las prácticas escolares de los educadores físicos. Perfiles Educativos. 2010; XXXII: 97-117.

26. Murcia N, Jaramillo L. Imaginarios del joven colombiano ante la clase de Educación física. Revista Latinoamericana de Ciencias sociales, niñez y juventud. 2006; 3(2): $1-28$

27. Díaz F, Hernández G. Estrategias docentes para un aprendizaje significativo. Una interpretación constructivista. En Constructivismo y aprendizaje significativo. $2^{\mathrm{a}}$ ed. Mc Graw Hill; 2002; 56.

28. Millán R. ¿Aprender? ¿Y por qué? Comunicación y humanidades. [Internet]. 2013. Disponible en [Consultado 201509 27] http://revistasum.umanizales.edu.co/ojs/index.php/filodepalabra/article/view/988/1104.

29. Hernández M. La evaluación del aprendizaje: ¿Estímulo o amenaza? Revista Iberoamericana de Educación. 2006; 1-8.

30. Murcia N, Jaramillo L. Imaginarios del joven colombiano ante la clase de Educación física. Revista Latinoamericana de Ciencias sociales, niñez y juventud. 2006; 3(2): 1-28.

31. López V. La evaluación como sinónimo de calificación. Implicaciones y efectos en la Educación y en la Formación del Profesorado. REIFOP. 2005; 8(4): 1-7.

32. Sequea E, Rodriguez Y. Evaluación formativa durante la práctica intensiva de docentes en educación integral. Revista Venezolana de Ciencias sociales. [Internet] 2006. Vol. 10(1): 255-269. [Consultado 201504 02] Disponible en http://www.redalyc.org/ articulo.oa?id=30910115.

33. Díaz F, Hernández G. Estrategias docentes para un aprendizaje significativo. Una interpretación constructivista. En Constructivismo y aprendizaje significativo. $2^{\mathrm{a}}$ ed. Mc Graw Hill; 2002; 56.

34. Millán R. ¿Aprender? ¿Y por qué? Comunicación y humanidades. [Internet]. 2013. Disponible en [Consultado 201509 27] http://revistasum.umanizales.edu.co/ojs/index.php/filodepalabra/article/view/988/1104.

35. Hernández M. La evaluación del aprendizaje: ¿Estímulo o amenaza? Revista Iberoamericana de Educación. 2006; 1-8.

36. Murcia N, Jaramillo L. Imaginarios del joven colombiano ante la clase de Educación física. Revista Latinoamericana de Ciencias sociales, niñez y juventud. 2006; 3(2): 1-28. 
37. López V. La evaluación como sinónimo de calificación. Implicaciones y efectos en la Educación y en la Formación del Profesorado. REIFOP. 2005; 8(4): 1-7.

38. Sequea E, Rodriguez Y. Evaluación formativa durante la práctica intensiva de docentes en educación integral. Revista Venezolana de Ciencias sociales. [Internet] 2006. Vol. 10(1): 255-269. [Consultado 201504 02] Disponible en http://www.redalyc.org/ articulo.oa?id=30910115.

Referencias totales citadas: 38

Referencias citadas correspondientes a la Rev Ib CC Act Fis Dep: 0 